\title{
Sub-ångström-resolution diffractive imaging of single nanocrystals
}

\author{
W. J. Huang, J. M. Zuo`, B. Jiang, K. W. Kwon and Moonsub Shim
}

\begin{abstract}
Diffractive imaging has the potential to succeed in structure determination of single nanoparticles using probes such as pulsed $X$-rays ${ }^{1}$ or medium-energy electrons ${ }^{2}$ where an atomicresolution imaging lens is not available and radiation damage can be remedied ${ }^{3}$. Although diffractive imaging has been demonstrated for particles ${ }^{4}$ and single cells $s^{5,6}$ at several nanometres in resolution, ultimately, atomic resolution is required to determine their three-dimensional structure. A major difficulty in atomic-resolution diffractive imaging is the loss of weak coherent scattering signals in recorded diffraction patterns. Here, we show that this can be overcome using information from low-resolution images. By combining information from both diffraction and imaging, we succeeded in phasing experimental electron diffraction patterns of individual CdS quantum dots at sub-ångström resolution. The low-resolution image provides the starting phase, realspace constraint, missing information in the central beam and essential marks for aligning the diffraction pattern, and diffraction provides high-resolution information. We show that for CdS nanocrystals, the improved image resolution enables determination of their atomic structures. As lowresolution images can be obtained from different sources, the technique developed here is general and provides a basis for imaging the three-dimensional atomic structure of single nanoparticles, where correct orientation of the recorded diffraction patterns is critical?
\end{abstract}

Diffractive imaging uses diffraction intensity and phase retrieval to form real-space images. By bypassing the need for imaging lenses and their associated aberrations, the resolution, in principle, is limited only by the amount of high-angle scattering ${ }^{8-10}$. For this reason, diffractive imaging has the potential to achieve atomic resolution for hard X-rays or other short-wavelength particles. For example, high-resolution diffractive imaging has been proposed for imaging biological molecules using ultrashort and extremely bright coherent X-ray pulses ${ }^{3}$. Ultrafast X-ray diffraction is intended to record diffraction patterns before the start of significant radiationinduced damage ${ }^{1,3}$. For electrons, the knock-on damage limits the number of images that can be recorded by high-resolution electron microscopes ${ }^{11}$. Imaging at below the knock-on damage threshold is critical for the determination of three-dimensional (3D) atomic structure. In such cases, diffractive imaging provides real-space information at resolutions that are not available otherwise.

The principle of diffractive imaging is based on coherent diffraction of an isolated object and recording of diffraction patterns at a spatial frequency $(f)$ smaller than the reciprocal of the object $\operatorname{size}^{12}(1 / S)$. The product of $(S f)^{-n}$, where $n$ is the dimension of the diffraction pattern, is called the oversampling ratio ${ }^{8}$. Oversampling increases the amount of information about the object recorded in the diffraction pattern, which is necessary for retrieving the phase required to form the image. Mathematically, a minimum oversampling ratio of 2 is required for diffractive imaging 9 . The smallest spatial frequency that can be recorded in an experiment is $1 / L$, where $L$ is the coherence length, which gives the maximum oversampling ratio $(L / S)^{n}$. However, the coherence length is only one of the limiting factors of information in experimentally recorded diffraction patterns; others include the background noise and missing intensities related to detector artefacts ${ }^{13,14}$. A major challenge of diffractive imaging is thus to overcome the limitation of experimental diffraction patterns. So far, successful examples of diffractive imaging all use strong signals at low spatial frequencies with resolution at several nanometres or larger ${ }^{4,14-16}$, with one exception of electron imaging of carbon nanotubes ${ }^{17}$.

To illustrate the challenge of atomic-resolution diffraction imaging of nanocrystals, we calculated the diffraction pattern of a model nanocrystal as shown in Fig. 1. The pattern shows strong diffraction spots that are associated with Bragg scatterings and also interference between different diffraction spots. There is a large difference in the intensity scale; the weak interference between Bragg spots is at least 2-3 orders of magnitude lower than the peak intensity of the diffraction spots. Figure 2 shows an experimental diffraction pattern of a single CdS quantum-dot diffraction pattern after background subtraction. The quantum dot observed here is about $7.8 \mathrm{~nm}$ in diameter. The central beam in this case is missing owing to the background subtraction and the loss of information caused by its strong intensities. Compared with the calculated diffraction pattern of Fig. 1, the strong diffraction peak and oscillations around the diffraction spots due to the finite size and shape of the quantum dot are clearly observed in the diffraction pattern. The weak interference intensities seen in the calculation are lost owing to the background noise. The detector itself has a dark noise of about 1 electron per pixel ${ }^{18}$. It is also clear that the amount of information in each diffraction spot decreases as the scattering angle increases. Although the amount of information lost may vary from experiment to experiment, in general, atomic-resolution diffractive imaging must overcome (1) the reduced information at high scattering angles due to the damping of atomic scattering factors, (2) the diffraction background noise and (3) the loss of information in the central beam ${ }^{13,14}$.

To achieve sub-ångström resolution from diffraction patterns with limited information, we have developed a modified phaseretrieval algorithm by incorporating information from an image (low resolution) and a diffraction pattern. We first use the lowspatial-frequency information contained in the low-resolution image to supplement the missing data in the central beam. We then 


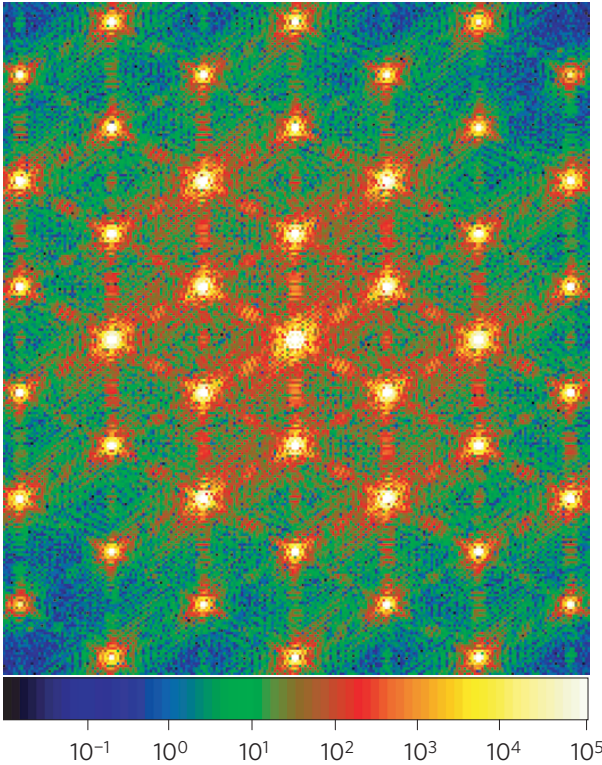

Figure 1 | Calculated [0001] diffraction pattern of a model CdS nanocrystal of hexagonal wurtzite structure and $9 \mathrm{~nm}$ in diameter at the pixel resolution of $\mathbf{0 . 0 0 6 7} \AA^{-\mathbf{1}}$. The diffraction pattern is shown on a log scale with a display range of $10^{-2}-10^{5}$ according to the colour bar shown below.

use the phase in the image to start the iteration. The image itself is used to obtain object support. A separate support in reciprocal space is used where we separate the diffraction pattern into three different regions of $G$ above or $B$ below the background noise level and $M$ where diffraction information is not available. We apply the diffraction intensity constraint in $G$. In region $B$, we place an upper limit of three times the background noise on the intensity, whereas in region $M$, the intensity is allowed to float. Iterative transformation is carried out to extend the phase to high-angle scattering recorded in diffraction patterns. The idea is similar to the widely used phase-extension technique in crystallography for extended periodic structures ${ }^{19,20}$. However, the method developed here is much more general and can be applied to non-periodic structures, including surfaces and interfaces and local defects. We demonstrate the power of our technique by imaging CdS quantum dots, which are one of most studied nanostructures. We selected CdS quantum dots for imaging because of their size-dependent optical properties ${ }^{21,22}$ and the importance of atomic structure on these properties ${ }^{23}$.

The experimental realization of our technique involves three separate steps of (1) diffraction pattern recording, (2) processing of experimental data and (3) iterative phase retrieval and image reconstruction. Experimental data processing involves (1) subtraction of diffraction background, (2) processing and scaling of the experimental low-resolution image to match the recorded diffraction pattern and (3) preparation of support masks for both real and reciprocal spaces. To record experimental diffraction patterns, we used the experimental set-up inside a transmission electron microscope (TEM) for a $200 \mathrm{kV}$ electron beam of about $40 \mathrm{~nm}$ in diameter with a lateral coherence length of about $35 \mathrm{~nm}$, as described before ${ }^{17}$. The experimental diffraction patterns reported here were recorded using an electron dose of $10^{6} \mathrm{e} \mathrm{nm}^{-2}$. At this dose, several diffraction patterns can be recorded without significant damage to the structure. The CdS quantum dots were supported by a substrate for diffraction. The use of a substrate removes the very challenging experimental problem associated with injecting nanoparticles into a diffraction chamber ${ }^{24}$, while

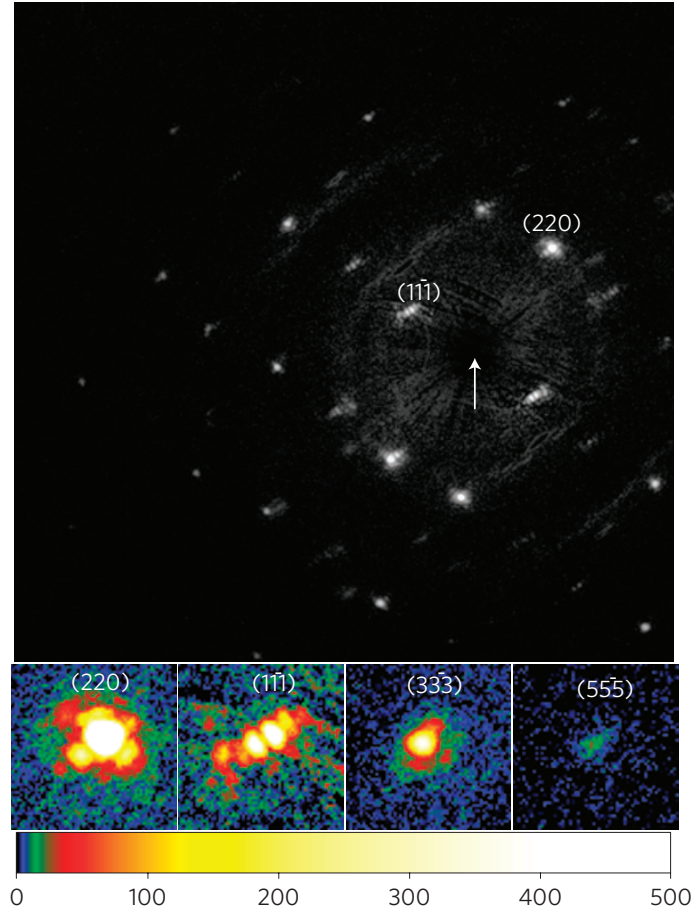

Figure 2 | Part of an electron diffraction pattern after background subtraction of a CdS nanocrystal of $7 \mathrm{~nm}$ in diameter in the [112] cubic orientation. The intensity distributions of selected diffraction spots are shown below. The index is based on the hexagonal wurtzite structure of $\mathrm{CdS}$. The colour scale is shown below.

the substrate introduces extra background diffraction intensities. We use ultrathin graphene or carbon nanotubes as the substrate to minimize the background intensity. We also use the mismatch between the graphene lattice and CdS nanocrystals to reduce the overlap of the two diffraction spots. Details about the preparation of CdS quantum dots, the substrate and the diffraction experiment are described in the Methods section. For background subtraction, two diffraction patterns were recorded: one with the quantum dot and one without it in a region close to the quantum dot. A cross-correlation analysis was carried out to align the background and particle diffraction patterns using rotation and shifts in the $x$ and $y$ directions. This was necessary in our experiment because of small mechanical misalignments introduced by the detector. An example of a CdS quantum-dot diffraction pattern after background subtraction is shown in Fig. 2. This diffraction pattern can be indexed as the [112] zone axis of the cubic spheralite structure of CdS. It is interesting to note that some of the diffraction spots are highly complex unlike the single diffraction peaks expected from ideal nanocrystals of Fig. 1.

Figure 3 compares the recorded electron image with the reconstructed higher-resolution image from the diffraction pattern shown in Fig. 2. The reconstructed image was averaged over 20 reconstructions; each started with a different set of constant noise that was added to the experimental low-resolution image. The low-resolution image was recorded using the same TEM used to record the diffraction pattern and was processed according to the procedures described in the Methods section. The same image was also used to estimate the object support that separates the object from the background. The details of the reconstruction are described in the Methods section.

The resolution improvement of the reconstructed image over the TEM image can be directly seen in the top of Fig. 3a,b. The improvement is further demonstrated in the power spectra of 
a

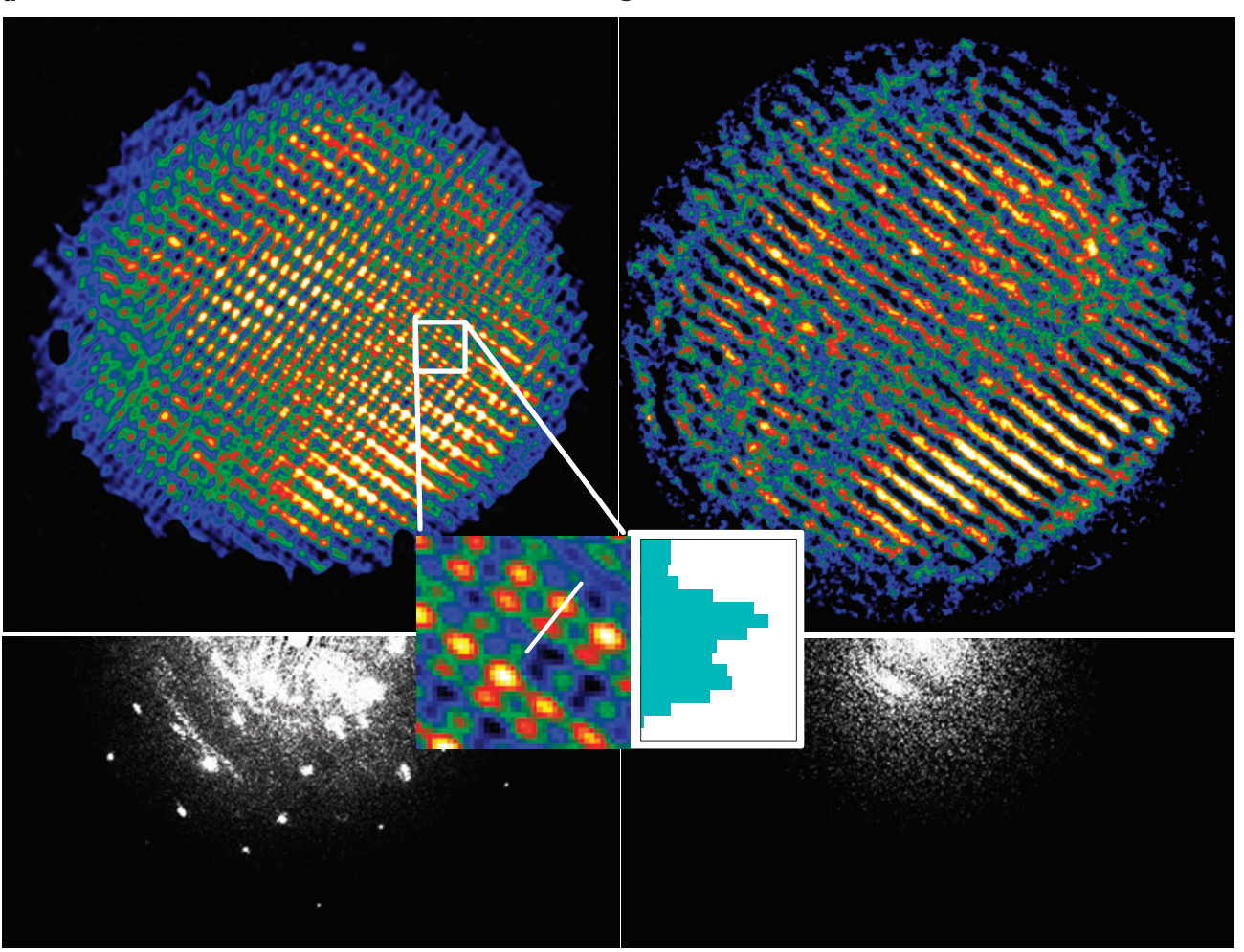

Figure 3 | Sub-ångström imaging of a CdS quantum dot of $\mathbf{7 ~ n m ~ i n ~ d i a m e t e r ~ a l o n g ~ t h e ~ c u b i c ~ C d S ~ c r y s t a l ~ [ 1 1 2 ] ~ o r i e n t a t i o n . ~ a , ~ T h e ~ r e c o n s t r u c t e d ~ i m a g e ~}$ using information from the starting image and the diffraction pattern of Fig. 2 (top) and its power spectrum (bottom). The inset shows a magnification of the outlined region. An intensity profile taken along the line shown in the inset demonstrates that the $0.84 \AA$ separation between the $\mathrm{Cd}$ and $\mathrm{S}$ atomic columns is well resolved and the lower peak can be attributed to $S$. b. The starting electron image with lattice fringe contrast (top) and its power spectrum (bottom).

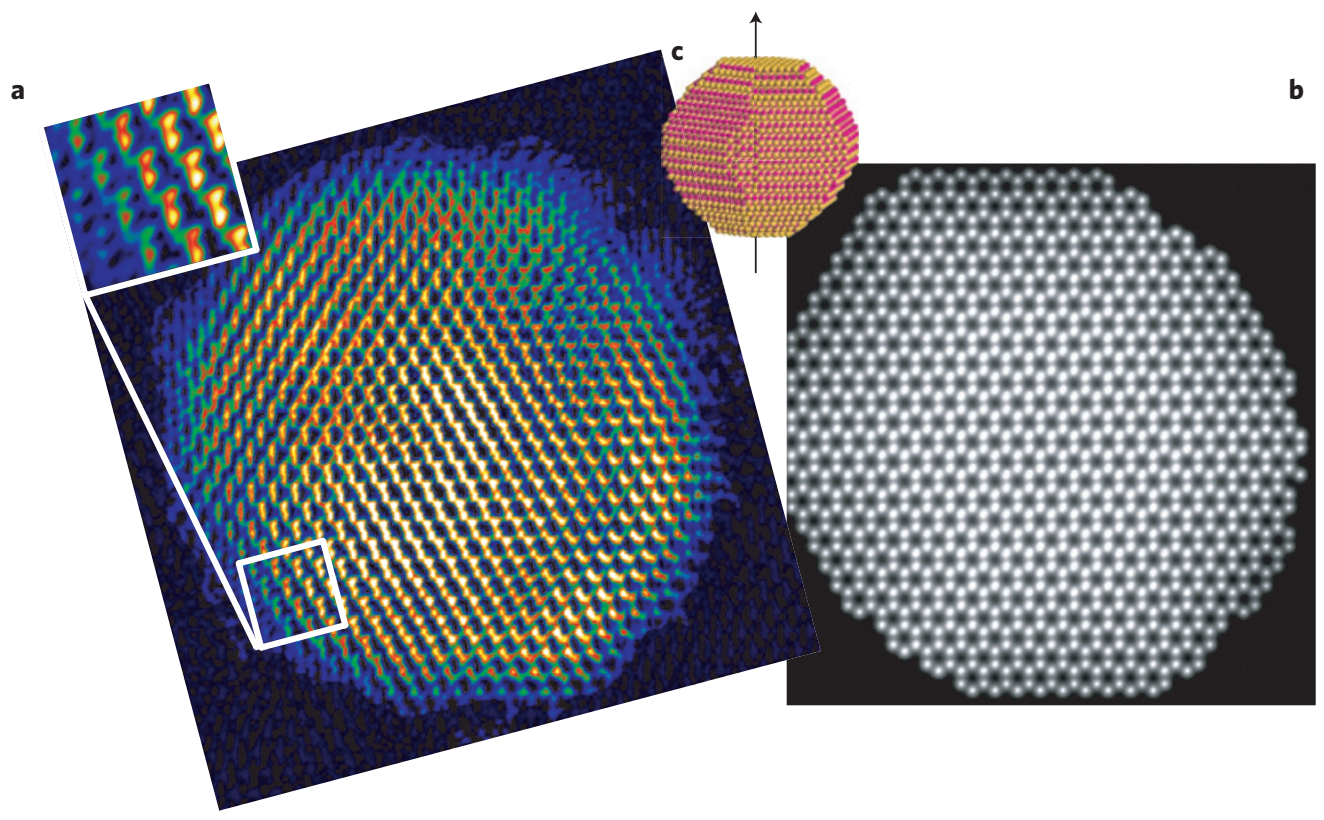

Figure 4 | A reconstructed image of a 9-nm-diameter CdS quantum dot along [0001]. a, Reconstructed image with improved resolution. $\mathbf{b}$, The projected potential of an atomic structure model constructed based on a. c. The estimated 3D model of the nanocrystal.

the images shown at the bottom of Fig. 3a,b. The information in the reconstructed image extends to the cubic $(62 \overline{4})$ reflection of $0.78 \AA d$-spacing. In comparison, only the $\pm(1 \overline{1} 1)$ cubic reflections of $3.3 \AA d$-spacing are present in the power spectrum of the starting image. Thus, the resolution improvement from the diffraction pattern is about a factor of 4 . The reconstructed image from the diffraction pattern shows clearly resolved atomic columns in areas near the highlighted region. At the orientation where the diffraction pattern was recorded, the smallest separation between the $\mathrm{Cd}$ and $\mathrm{S}$ atomic columns is $0.84 \AA$. This is 
clearly resolved in Fig. 3. Asymmetric peaks are also seen in the reconstructed image from the intensity profile, taken across a pair of atoms, as shown in the inset of Fig. 3. In the weakphase-object approximation, the real part of the complex object is proportional to the object potential and therefore a larger peak is expected for Cd than S. Using this information, the crystal polarity can be directly determined from the reconstructed image. Interestingly, both the low-resolution starting image and the reconstructed image indicate an interface present within the particle (see Fig. 3). The lattices on the two sides of the boundary have a very small misorientation, as seen from the change in the contrast of the atomic columns. The lattice is also shifted across the boundary. The shift, measured directly from the reconstructed image, is $1.6 \AA$. The small-angle boundary is also consistent with split Bragg peaks for some reflections, most notably the $\pm(11 \overline{1})$ and $\pm(22 \overline{2})$, but not others such as (220) in the diffraction pattern, as shown in Fig. 2, because the lattice shift occurs only for the $\pm(11 \overline{1})$ lattice. The reconstructed image thus provides a direct interpretation of the complex diffraction pattern here.

Figure 4 shows a case where the improved image contrast in the reconstructed image enables determination of the crystal facets of the CdS quantum dot. The starting image and the diffraction pattern were recorded along the [0001] hexagonal zone axis. In this projection, the $\{10 \overline{1} 0\}$ facets of a hexagonal crystal can be determined directly from a high-resolution image. The resolution of the as-recorded high-resolution electron microscope (HREM) image contains the lattice fringes of $\{10 \overline{1} 0\}$ and $\{11 \overline{2} 0\}$ of spacing $3.58 \AA$ and $2.07 \AA$ respectively. The information in the reconstructed image of Fig. 4 extends to about $(40 \overline{4} 0)$ with a $d$-spacing of $0.90 \AA$. At this resolution, the projected hexagonal network of $\mathrm{Cd} / \mathrm{S}$ atomic columns is clearly resolved. The image contrast is also significantly improved compared with that of images obtained by aberration-corrected scanning transmission electron microscopy using an annular dark-field detector ${ }^{25}$. The contrast improvement comes from a special advantage of diffractive imaging; the support can be removed from the image as long as its diffraction does not interfere with the object. The improved contrast enables clear identification of the edges of the nanocrystal. Using this information, we constructed a nanocrystal model, which is shown in Fig. $4 \mathrm{~b}$ as the projected potential. The facets identified include those belonging to $\{10 \overline{1} 0\}$ and $\{1 \overline{1} 0\}$. Rounded corners can also be seen in the image. The estimated $3 \mathrm{D}$ structure of the nanocrystal is shown in Fig. 4c. This 3D model includes the $\{10 \overline{1} 1\}$ facets, which was suggested from the intensity profile of the reconstructed image. However, a complete determination of the 3D structure was not possible in this case. The recorded diffraction pattern suggests that the nanocrystal is slightly misoriented away from the zone axis. By modelling the diffraction pattern, we oriented the nanocrystal model in a similar way as the experimental image, which is shown in Fig. $4 \mathrm{~b}$. In this orientation, the atomic columns are slightly rotated and elongated, which closely resembles the experimentally observed contrast as seen in the inset of Fig. 4a. However, it must be pointed out that whereas the atomic columns show similar contrast in the nanocrystal model, the experimental image shows slight deviations across the nanocrystal suggesting a less perfect structure than the ideal nanocrystal model.

\section{Methods}

The CdS quantum dots studied here were synthesized using the following procedures. A solution containing $300 \mathrm{mg}$ of trioctylphosphine oxide, $315 \mathrm{mg}$ 1,2-hexadecanediol and $10 \mathrm{ml}$ of octyl ether was vacuum degassed at $100^{\circ} \mathrm{C}$ for $30 \mathrm{~min}$. Sulphur powder $(15 \mathrm{mg})$ was added at $100^{\circ} \mathrm{C}$ under $\mathrm{N}_{2}$ and stirred for $5 \mathrm{~min}$. After cooling to $80^{\circ} \mathrm{C}$, cadmium acetylacetonate $(150 \mathrm{mg})$ was added and stirred for $10 \mathrm{~min}$. The reaction mixture was heated to $280^{\circ} \mathrm{C}$ and annealed for $30 \mathrm{~min}$. The final CdS nanocrystals were precipitated with ethanol, centrifuged to remove excess capping molecules and redissolved in chloroform. The CdS quantum dots were supported on ultrathin graphene sheets (2-3 layers) or carbon nanotubes for electron diffraction. Diffraction patterns were recorded at the camera length of $80 \mathrm{~cm}$ using imaging plates with a pixel size of $25 \mu \mathrm{m}$. A separate diffraction pattern was also recorded without the quantum dot. This diffraction pattern is used as the background and subtracted from the particle diffraction pattern. The diffraction peaks of the CdS quantum dot range from a few to several thousand counts recorded on the imaging plates with a conversion rate about 0.8 counts per incident electron. In phasing the diffraction patterns in this letter, we used a $2,048 \times 2,048$ array with a field of view of $400 \AA$. The experimental diffraction pattern was binned by a factor of 2 and centred inside the array. The centring was carried out by cross-correlation between strong diffraction spots between $\pm g$ pairs.

An electron image with the background intensity normalized to 1 recorded from the same area where the diffraction pattern was taken was used as the so-called 'low-resolution image' (LRI) for subsequent discussion. We first estimate the projected object potential from the electron LRI based on the weak-phase-object approximation ${ }^{26}$ :

$$
V^{\exp }(x, y)=V(x, y) \otimes H(x, y)+N(x, y) \simeq[I(x, y)-1] / 2 \sigma+N(x, y),
$$

where $V(x, y)$ is the projected object potential, $\sigma$ is the electron interaction constant and $H$ is the microscope resolution function. $N$ is a small constant noise that we add to the image. This noise provides the random starting phase for high-angle scattering in the diffraction pattern beyond the information limit of the LRI. The estimated potential $V^{\exp }(x, y)$ was then rotated and scaled to match the recorded diffraction pattern by optimizing the cross-correlation coefficient between the power spectrum of the potential $\left(I^{\mathrm{P}}\right)$ and the diffraction pattern $\left(I^{\mathrm{D}}\right)$. The cross-correlation was calculated only for diffraction peaks that belong to both patterns.

The object support was obtained from the estimated potential by applying a threshold and converting it to a binary image using the published procedures ${ }^{27}$. The same procedures were applied to the diffraction pattern to obtain the diffraction mask where the threshold is the background noise level. We set the mask to three different levels, $G, M$ and $B$, as described in the text. The two supports obtained were used throughout the phase-retrieval procedure. The support obtained from the image is also tight enough to meet the requirement for complex object reconstruction ${ }^{28,29}$.

We used Fienup's hybrid input-output algorithm for phase retrieval ${ }^{30}$. The algorithm was modified by imposing a constraint on the real-space image intensity so that the potential, $\tilde{V}_{n}(x, y)$, obtained during the initial stage of iterations agrees with the estimated object potential, $V^{\exp }(x, y)$, at a reduced resolution:

$$
V^{\exp }(x, y) \otimes h_{1}(x, y) \approx \tilde{V}_{n}(x, y) \otimes h_{2}(x, y) .
$$

Here, $h_{1}$ and $h_{2}$ are selected to account for the difference in resolutions of the two potentials. In the reciprocal space, we replace the amplitudes of the Fourier transform according to

$$
F(u, v)= \begin{cases}F(u, v) \cdot \sqrt{I^{\mathrm{D}}(u, v)} /|F(u, v)| & \text { if }(u, v) \in G \\ F(u, v) \cdot \alpha \cdot \min \left(\sqrt{I^{\mathrm{D}}(u, v)}, \sqrt{3 \sigma}\right) /|F(u, v)| & \text { else if }(u, v) \in B \\ F(u, v) & \text { if }(u, v) \in M,\end{cases}
$$

where $G, B$ and $M$ mark different regions in the reciprocal space mask, as described before. $I^{\mathrm{D}}$ is the experimental diffraction intensity, $\alpha$ is a fractional number that was used to reduce the effect of the background intensity on the reconstructed image (we used $\alpha=0.3$ here) and $\sigma$ is the standard deviation of the background intensity. The maximum of $3 \sigma$ is imposed on the background intensity to remove artefacts from background subtraction. The image intensity constraint was used only in the initial stage of phase retrieval. Afterward, only the reciprocal space constraint is applied. Finally, the real-object constraint was relaxed and the complex wavefunction was reconstructed in the last step. A typical reconstruction takes about 20 iterations with the image constraint followed by $50-100$ iterations with the real-object constraint. The final step with complex object reconstruction takes an extra 50-100 iterations. We use the $R$-factor to monitor the phasing process. With the real-object constraint, the $R$-factor comes down to about a few per cent. The $R$-factor drops rapidly once the real constraint is relaxed. In a typical reconstructed image, the imaginary part is much less than the real part at $\sim 1 / 20$. The residual noise in the diffraction pattern introduces a small intensity difference in reconstructed images using different starting, random, phases. To remove these fluctuations, we averaged about 20 images reconstructed with different sets of random noise. 
Received 29 September 2008; accepted 25 November 2008; published online 21 December 2008

\section{References}

1. Chapman, H. N. et al. Femtosecond diffractive imaging with a soft-X-ray free-electron laser. Nature Phys. 2, 839-843 (2006).

2. Kamimura, O. et al. Diffraction microscopy using $20 \mathrm{kV}$ electron beam for multiwall carbon nanotubes. Appl. Phys. Lett. 92, 024106 (2008).

3. Neutze, R., Wouts, R., van der Spoel, D., Weckert, E. \& Hajdu, J. Potential for biomolecular imaging with femtosecond X-ray pulses. Nature 406, 752-757 (2000).

4. Miao, J. W. et al. Three-dimensional $\mathrm{GaN}-\mathrm{Ga}_{2} \mathrm{O}_{3}$ core shell structure revealed by X-ray diffraction microscopy. Phys. Rev. Lett. 97, 215503 (2006).

5. Shapiro, D. et al. Biological imaging by soft x-ray diffraction microscopy. Proc. Natl Acad. Sci. USA 102, 15343-15346 (2005).

6. Miao, J. W. et al. Imaging whole Escherichia coli bacteria by using single-particle x-ray diffraction. Proc. Natl Acad. Sci. USA 100, 110-112 (2003).

7. Shneerson, V. L., Ourmazd, A. \& Saldin, D. K. Crystallography without crystals. I. The common-line method for assembling a three-dimensional diffraction volume from single-particle scattering. Acta Crystallogr. A 64, 303-315 (2008).

8. Miao, J. W., Charalambous, P., Kirz, J. \& Sayre, D. Extending the methodology of X-ray crystallography to allow imaging of micrometre-sized non-crystalline specimens. Nature 400, 342-344 (1999).

9. Bates, R. H. T. Fourier phase problems are uniquely solvable in more than one dimension. 1. Underlying theory. Optik 61, 247-262 (1982).

10. Abbey, B. et al. Keyhole coherent diffractive imaging. Nature Phys. 4, 394-398 (2008)

11. Reimer, L. Transmission Electron Microscopy (Springer, 1997).

12. Sayre, D., Chapman, H. N. \& Miao, J. On the extendibility of X-ray crystallography to noncrystals. Acta Crystallogr. A 54, 232-239 (1998).

13. Marchesini, S. et al. X-ray image reconstruction from a diffraction pattern alone. Phys. Rev. B 68, 140101 (2003).

14. Thibault, P., Elser, V., Jacobsen, C., Shapiro, D. \& Sayre, D. Reconstruction of a yeast cell from X-ray diffraction data. Acta Crystallogr. A 62, 248-261 (2006).

15. Chapman, H. N. et al. High-resolution ab initio three-dimensional $\mathrm{x}$-ray diffraction microscopy. J. Opt. Soc. Am. A 23, 1179-1200 (2006).

16. Pfeifer, M. A., Williams, G. J., Vartanyants, I. A., Harder, R. \& Robinson, I. K. Three-dimensional mapping of a deformation field inside a nanocrystal. Nature 442, 63-66 (2006).

17. Zuo, J. M., Vartanyants, I., Gao, M., Zhang, R. \& Nagahara, L. A. Atomic resolution imaging of a carbon nanotube from diffraction intensities. Science 300, 1419-1421 (2003).

18. Zuo, J. M. Electron detection characteristics of a slow-scan CCD camera, imaging plates and film, and electron image restoration. Microsc. Res. Tech. 49, 245-268 (2000).
19. Fan, H. F., Zhong, Z. Y., Zheng, C. D. \& Li, F. H. Image-processing in high-resolution electron-microscopy using the direct method. I. Phase extension. Acta Crystallogr. A 41, 163-165 (1985).

20. Ishizuka, K., Miyazaki, M. \& Uyeda, N. Improvement of electron-microscope images by the direct phasing method. Acta Crystallogr. A 38, 408-413 (1982).

21. Brus, L. Quantum crystallites and nonlinear optics. Appl. Phys. A 53 , 465-474 (1991).

22. Alivisatos, A. P. Semiconductor clusters, nanocrystals, and quantum dots. Science 271, 933-937 (1996).

23. Rosenthal, S. J., McBride, J., Pennycook, S. J. \& Feldman, L. C. Synthesis, surface studies, composition and structural characterization of CdSe, core/shell and biologically active nanocrystals. Surf. Sci. Rep. 62, 111-157 (2007).

24. Spence, J. C. H. et al. Diffraction and imaging from a beam of laser-aligned proteins: Resolution limits. Acta Crystallogr. A 61, 237-245 (2005).

25. Nellist, P. D. et al. Direct sub-angstrom imaging of a crystal lattice. Science 305, 1741-1741 (2004)

26. Spence, J. C. H. High-Resolution Electron Microscopy (Oxford Univ. Press, 2003).

27. Huang, W. J., Jiang, B., Sun, R. S. \& Zuo, J. M. Towards sub-A atomic resolution electron diffraction imaging of metallic nanoclusters: A simulation study of experimental parameters and reconstruction algorithms. Ultramicroscopy 107, 1159-1170 (2007).

28. Fienup, J. R. Reconstruction of a complex-valued object from the modulus of its Fourier transform using a support constraint. J. Opt. Soc. Am. 6 , 118 (1987).

29. Spence, J. C. H., Weierstall, U. \& Howells, M. Phase recovery and lensless imaging by iterative methods in optical, X-ray and electron diffraction. Phil. Trans. R. Soc. Lond. 360, 875-895 (2002).

30. Fienup, J. R. Phase retrieval algorithms-a comparison. Appl. Opt. 21, 2758-2769 (1982)

\section{Acknowledgements}

This material is based on work supported by the US Department of Energy, Division of Materials Sciences under Award No. DEFG02-01ER45923 and No. DEFG02-91ER45439, through the Frederick Seitz Materials Research Laboratory at the University of Illinois at Urbana-Champaign. In addition, M.S. acknowledges support by the ACS PRF (grant No. 46443-AC10) and W.J.H. acknowledges support by the NSF (DMR 0449790) on work related to the preparation of electron diffraction samples.

\section{Author contributions}

W.J.H., K.W.K. and M.S. contributed to the preparation of CdS quantum dots for electron diffraction. J.M.Z., B.J. and W.J.H. contributed to the development of electron diffractive imaging methods and experimental data processing.

\section{Additional information}

Reprints and permissions information is available online at http://npg.nature.com/ reprintsandpermissions. Correspondence and requests for materials should be addressed to J.M.Z. 